\title{
Energy Aware Task Allocation with Unequal Clustering in WSN
}

\author{
DeveshPratap Singh \\ Dept of CSE \\ Graphic Era University \\ Dehradun, India
}

\author{
R.H. Goudar \\ Dept of CNE \\ VTU \\ Belgaum, India
}

\author{
Bhaskar Pant \\ Dept of IT \\ Graphic Era University \\ Dehradun, India
}

\begin{abstract}
Extending the lifetime of the wireless sensor network has been aimed by several research efforts. Distributed networks provide a viable solution to increase the lifetime of WSN. It does not assign the heavy task to a single node, which otherwise can result in the loss of connectivity, fast depletion of the battery of the single node and reallocation of the tasks. Task allocation in WSN play a major role in extending the lifetime. This paper propose a method(ETAUC) Energy aware task allocation with unequal clustering in WSN that provide the task allocation among clusters according to their strength of the battery, it helps in balancing the work in network and avoids the condition, in which some clusters finishes quickly and other clusters have plenty of battery power. The proposed method also circumlocutes the reassignment of the task; which has been partially completed by the finished cluster. The wireless sensor networks are deployed in the area of variant environmental features so we have also focused here on providing the relation between the temperature and the battery power of the sensor node. The simulation results shows that our proposed method increase the throughput $32.51 \%$.
\end{abstract}

\section{Keywords}

WSN, Coverage lifetime, Task allocation, Clustering.

\section{INTRODUCTION}

Network of large number of sensor nodes can be used for military surveillance, habitat monitoring, vehicle tracking and infrastructure monitoring [1],[2]. The sensor nodes initially contain a limited amount of battery; the battery of the sensor cannot be recharged due to the application area of the wireless sensor network. Because of this reason many researchers have focused on the utilization of battery in WSN. Cluster based tracking sensor networks comes under the category of collaborative applications, in which many tasks completed in cooperative manner[3],[4],[5],[6],[7]. The energy conservation analysis is required for sensor nodes because these nodes contain fewer amounts of battery capacities operating in tactical areas [8],[9], [10],[11]. As the wireless sensor network contains many clusters, so it is possible that the depletion rate of the battery power of one cluster is more as compared to other clusters, in general many clusters reside in the network with a sufficient amount of the battery with no connectivity, so we cannot apply these clusters to do any task. The Temperature affects the depletion rate of the battery power[12].

Clustering is an important method to implement the hierarchical topology, which increases the scalability and lifetime of the sensor network. Each cluster consists of a cluster head node and many member nodes. The cluster head performs the task of distribution and aggregation, so the battery utilization of cluster head becomes high as compared to the other nodes present in the cluster. To compensate the maximum utilization of the battery by cluster head, the rotation can be done in cluster for appointing the cluster head within the cluster.

The distributed computing is important for increasing the network lifetime, network processing power [13],[14],[15],[16]. However, there is importance for task allocation for boosting the efficiency of the network. WSN is a event driven network, so in this each node contain different amount of battery.

In this work, ETAUC, An Energy Aware Task Distribution with unequal Clustering in WSN, is proposed. ETAUC's objective is to provide balanced task allocation within the network, so that the allocated task becomes completed within the lifetime of the node/cluster. It considers the effect of temperature in the depletion rate of the battery; according to the depletion rate it assigns the task to the nodes present in the cluster.

This paper is organized as: section II discusses the closely related works, section III explains the problem description, section IV presents the ETAUC algorithm, section V shows the simulation results, section VI concludes with a brief summary.

\section{RELATED WORK}

A large amount of studies have been done on the reduction of the utilization of battery power and task allocation in WSN by using the different technique [10], [11], [17], [20].

Y. Yu and V. K. Prasanna have proposed an algorithm for achieving the energy balanced

task allocation in a single-hop cluster[21]. They have proposed the following algorithm for task allocation:

Step 1: cluster of tasks are formed by removing the heaviest communication activities for reducing the overall execution time of the tasks.

Step 2: Cluster of tasks are assigned to the sensor which has minimum norm-energy.

Step 3: In this step several iterations are used for achieving the energy balancing by reducing the energy of the critical node.

In this algorithm, the tasks are assigned to the nodes which have less amount of battery; it has a negative impact on the overall throughput of the system because the nodes with less 
amount of battery power early come to end. The proposed ETAUC algorithm assigns the percentage of task according to the remaining energy percentage; it assures that the allocated proportion of task should be completed by that particular node.

BEATA algorithm that is given in [22] and [23], consider the collaborative applications. It maintains an energy adaptive window. All the nodes are sorted according to the finish time of a task. The nodes in this list that utilizes less amount of battery are chosen for assigning the next task. Unlike the ETAUC, it does not provide the energy balancing in task allocation because the nodes with higher processing power have more chance to present in the energy adaptive window, and hence, more chance to get the next task. As a result the battery power of nodes with higher processing power diffuses early.

J. Zhu, J. Li and H. Gao, have proposed a task allocation algorithm for heterogeneous sensor network with the effort to reduce the energy consumption in the network while meeting the deadline, they have followed the divide and conquer paradigm[24]. The algorithm contains four phases:

i) Task Partition

ii) Deadline distribution

iii) Scheduling

iv) Rescheduling

The algorithm considered the task either synchronization task or simple task. The deadline is distributed among the synchronization task present in the task set. In scheduling phase the task is assigned to the node, that consumes the less energy and fulfill the deadline In this algorithm it is possible that energy is hugely sacrificed.

S.Giannecchini et al.[25] proposed a algorithm (CoRAI) for assigning the network resources to tasks. They have not discussed about the energy consumption and allocation of tasks to sensor nodes.

P. Basu, W. Ke, and T. D. C. Little described a TCP oriented task mapping algorithms with respect to an average hopcount[26]. They have not considered the heavy load in the given algorithm.

\section{PROBLEM FORMULATION}

\subsection{System Models}

This section presents different mathematical models for task assignment framework. We will describe here, Network system model, Task Model, cluster construction phase etc.

\subsubsection{Network system model}

The network system,in most general way consist of a set of clusters e.g. $\mathrm{N}=\left\{\mathrm{c}_{1}, \mathrm{c}_{2}, \mathrm{c}_{3}, \mathrm{c}_{4} \ldots \ldots \ldots \ldots \mathrm{c}_{\mathrm{n}}\right\}$. Each cluster is a collection of, for exampleC $=\left\{n_{1}, n_{2}, n_{3}, n_{4} \ldots \ldots \ldots n_{t}\right\}$ sensor nodes. The number of nodes in clusters is variant and each node contains a different amount of battery power with equal power of computation efficiency. The consumption rate of the battery power of the node is measured in Joules per unit time. Each node in the cluster contains different amount of battery power for example $B=\left\{b_{1}, b_{2}, b_{3}, b_{4} \ldots \ldots \ldots b_{t}\right\}$. Total energy of the cluster is represented as $B_{c}=\sum_{i=1}^{t} b_{i}$. The network can be represented as the graph of nodes, in upper layer the clusters are connected by the point to point link and in lower layer; nodes are connected by the link. The link $X_{\mathrm{uv}}$ means the cluster u can communicate with the cluster v. The graph for upper layer $\left(l_{1}\right)$ can be modeled $\operatorname{asG}_{l_{1}}=\{N, X\}$, whereX $=\left\{\mathrm{X}_{12}, \mathrm{X}_{34}, \mathrm{X}_{35} \ldots \ldots \ldots \mathrm{X}_{\mathrm{pn}}\right\}$. The edge(link) $\mathrm{Y}_{\mathrm{ab}}$ represents that the node a can directly communicate with node b. The graph for lower layer $\left(l_{2}\right)$ is modeled $\operatorname{asG}_{l_{2}}=\{C, Y\}$, where $\mathrm{Y}$ is a set $\left\{\mathrm{Y}_{12}, \mathrm{Y}_{34}, \mathrm{Y}_{35} \ldots \ldots \ldots \mathrm{Y}_{\mathrm{qt}}\right\}$. An allocation matrix(A) $m \times n$ shows the amount of task assigned to a particular cluster. If an entry $\left(\mathrm{A}_{\mathrm{ij}}\right)=0$ is present in the allocation matrix, it shows that part of task $\mathrm{i}$ is not allocated to cluster $\mathrm{j}$ and if $\mathrm{A}_{\mathrm{ij}}=\mathrm{x}$, shows that $\mathrm{x}$ percent task of task $\mathrm{i}$ is assigned to the cluster $\mathrm{j}$.

\subsubsection{Task model}

An application that can be divided into multiple tasks, can be modeled by DAG[27]. DAG is represented in this paper as $\mathrm{G}=$ $\{\mathrm{T}, \mathrm{E}\}$, where $\mathrm{T}=\left\{t_{1}, t_{2}, t_{3} \ldots \ldots \ldots t_{n}\right\}$ represent a set of tasks in a particular application. The set $\left(\mathrm{t}_{\mathrm{i}}, \mathrm{t}_{\mathrm{j}}\right) \varepsilon \mathrm{E}$, represent that there is a directed edge from node $t_{i}$ to $t_{j}$. Each cluster has a cluster head $(\mathrm{H})$ that reside at the root node of the DAG. The $\mathrm{H}$ collects the result of the complete application from the different nodes in the DAG. Note that our ETAUC method assumes that the communication cost for sending the message between the nodes is $\mathrm{C}_{\mathrm{m}}$. if the packet size is given as $\mathrm{K}$ and the link capacity is given as $\mathrm{L}$, then the communication cost becomes $\mathrm{K} / \mathrm{L}$. The time to complete the task is given by the set $\mathrm{T}=\left\{s_{i_{1}}, s_{i_{2}}, s_{i_{3}} \ldots \ldots \ldots \ldots s_{i_{t}}\right\}$.

\subsubsection{Cluster construction phase}

For the constructing cluster we have applied the unsupervised learning. We have collected the data from [19]. The original dataset contains 8 fields as date, time, epoch, moteid, temperature, humidity, light, voltage. We have preprocessed the data and taken the fields of temperature and voltage, and applied the K-means clustering for constructing the clusters. Initially, we have defined the number of clusters as 2 .from the result we have got that for 2 clusters the density is higher at outer region of the clusters. We have increased the clusters from 2 to 3,3 to 4 and 4 to 5 . As we have taken 5 clusters the outcome results in the equal density of the clusters. Below figure 1 shows the density of the data.

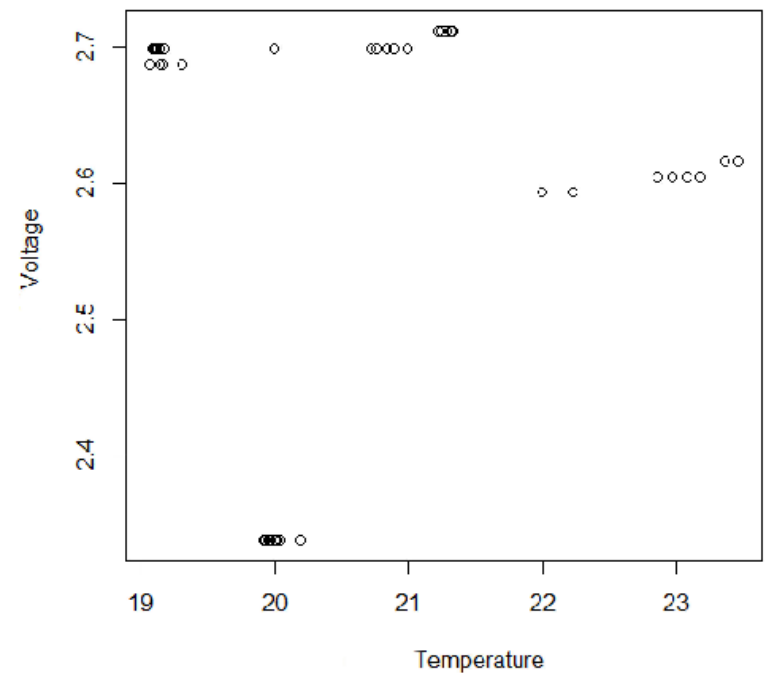

Fig. 1 Density Of Data Distribution 


\subsubsection{Task distribution model}

If the application is assigned to a particular cluster, the different tasks constitute the application are allocated to different nodes that present in this cluster according to their remaining battery power $\left(R_{b}\right)$. The percentage of task $t_{i}$ can be assigned to the node with remaining battery power $\mathrm{R}_{\mathrm{bi}}$ is given ast $_{\mathrm{i}} \times \frac{R_{b i}}{100}$. On the basis of remaining battery the tasks are assigned inside the cluster, then the partial completion of the assigned task to a node becomes negligible. The assignment of the task to the nodes of different level is decided by the upper level. E.g. the nodes at level $\mathrm{i}$ are $\mathrm{n}_{1}, \mathrm{n}_{2} ; \mathrm{n}_{1}$ contains $\mathrm{x} \%$ of task and $\mathrm{n}_{2}$ contains $\mathrm{y} \%$ of task assigned by the node present in level $i-1$, suppose that the nodes on level $i+1$ are $\mathrm{n}_{3}, \mathrm{n}_{4}$ and $\mathrm{E}$ is given as $\left\{\left(\mathrm{n}_{1}, \mathrm{n}_{3}\right),\left(\mathrm{n}_{1}, \mathrm{n}_{4}\right)\right\}$. Now the division of $\mathrm{x} \%$ of task is decided according to the remaining battery of the nodes $\mathrm{n}_{3}\left(\mathrm{R}_{\mathrm{bn} 3}\right)$ and $\mathrm{n}_{4}\left(\mathrm{R}_{\mathrm{bn} 4}\right)$ asx $\times \frac{\mathrm{R}_{\mathrm{bn} 3}}{100}$ andx $\times \frac{\mathrm{R}_{\mathrm{bn}_{4}}}{100}$ respectively. The below figure 2 shows the task distribution process.

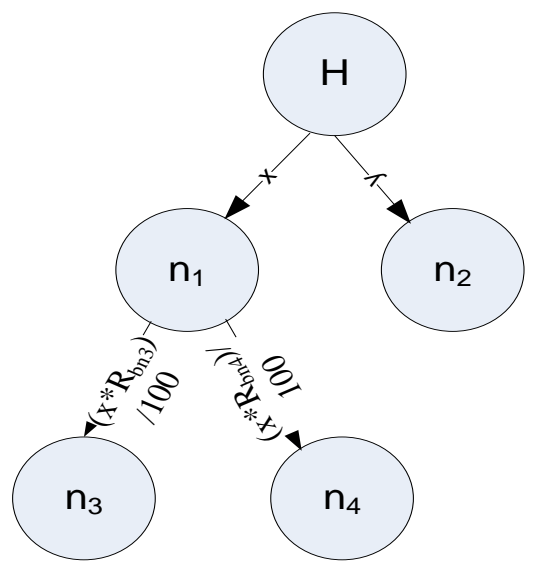

Fig. 2 Task Distribution Process

\subsubsection{Remaining battery model}

The remaining battery of the network is represented as the set

$\mathrm{B}_{\mathrm{N}}=\left\{\mathrm{R}_{\mathrm{BC}}^{1}, \mathrm{R}_{\mathrm{BC}}^{2}, \mathrm{R}_{\mathrm{BC}}^{3}, \mathrm{R}_{\mathrm{BC}}^{4} \ldots \ldots \ldots \ldots \mathrm{R}_{\mathrm{BC}}^{\mathrm{N}}\right\}$. The remaining battery of the network is given as $R_{B N}=\sum_{i=1}^{n} R_{B C}^{i}$. The Cluster's battery can be represented by the set as $\mathrm{B}=$ $\left\{B_{1}, B_{2}, B_{3}, B_{4} \ldots \ldots \ldots \ldots B_{t}\right\}$. Here, nodes of level, $i$ informs about their remaining battery to their parent level $\mathrm{i}-1$. The remaining battery of the level $i$ is calculated as $R_{B}^{\text {level } i}=$ $\sum_{\mathrm{k}=1}^{\mathrm{l}} \mathrm{R}_{\mathrm{B}}^{\mathrm{k}}$, where $\mathrm{k}=1,2,3,4 \ldots \ldots \ldots$. where, total number of nodes at level $\mathrm{i}$ are 1 . The remaining battery at each node at leveli, $R_{\mathbf{B} \text { node }}^{\text {level } i}$ is $\quad$ calculated as

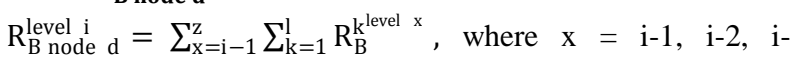
$3, \ldots \ldots \ldots \mathrm{z}$ and $\mathrm{z}$ is the level of the leaf node. At last, the cluster head, $\mathrm{H}$ calculates the remaining battery of the cluster after collecting the remaining battery information of their child nodes.

\subsubsection{Automatic Task's Result Collection Model}

The cluster head of each cluster collects the result send by the nodes $\mathrm{C}=\left\{\mathrm{n}_{1}, \mathrm{n}_{2}, \mathrm{n}_{3}, \ldots \ldots \ldots \ldots \ldots \mathrm{n}_{\mathrm{t}}\right\}$. The nodes in this model cannot wait for other nodes to complete the assigned task. When, any node $\mathrm{n}_{\mathrm{i}}$ completes its assigned task, it send the result to their parent at level $\mathrm{i}-1$. The parent node $\mathrm{n}_{\mathrm{p}}$ at level $\mathrm{i}$ -
1 collects the result from their child nodes and assemble the result that is provided by their child into one result. For example, The nodes at level $i$ are $n_{\mathrm{i} 1}, \mathrm{n}_{\mathrm{i} 2}, \mathrm{n}_{\mathrm{i} 3}$ and $\mathrm{n}_{\mathrm{i} 4}$, the parent of these nodes at level i-1 is $n_{p}$. The parent node receives the result from the nodes $\mathrm{n}_{\mathrm{i} 1}, \mathrm{n}_{\mathrm{i} 2}, \mathrm{n}_{\mathrm{i} 3}$ and $\mathrm{n}_{\mathrm{i} 4}$ assemble these result into one or some message(result) asResult $\mathrm{n}_{\mathrm{np}}, \mathrm{n}_{\mathrm{i}_{2},}, \mathrm{n}_{\mathrm{i}_{3},}, \mathrm{n}_{\mathrm{i}_{4}}=$ Result $_{\mathrm{n}_{\mathrm{i}_{1}}}||$ Result $_{\mathrm{n}_{\mathrm{i}_{2}}}||$ Result $_{\mathrm{n}_{\mathrm{i}_{3}}}||$ Result $_{\mathrm{n}_{\mathrm{i}_{4}}}$. The node $\mathrm{n}_{\mathrm{p}}$ forwarded the resultsResult $\mathrm{t}_{\mathrm{np}} \mathrm{n}_{\mathrm{i}_{1}}, \mathrm{n}_{\mathrm{i}_{2}}, \mathrm{n}_{\mathrm{i}_{3}}, \mathrm{n}_{\mathrm{i}_{4}} \|$ Result ${ }_{n p}$ to their parent at level i-2. In this way the cluster head $(\mathrm{H})$ can collect all the result generated from the C. Here, in our model, it is assumed that there is no need for any node that is present inside any cluster to send the incompleted assignment to their parent node at upper level. The base station in WSN collects the result from each cluster present in the network. The procedure for Task Result collection is shown in the figure 3.

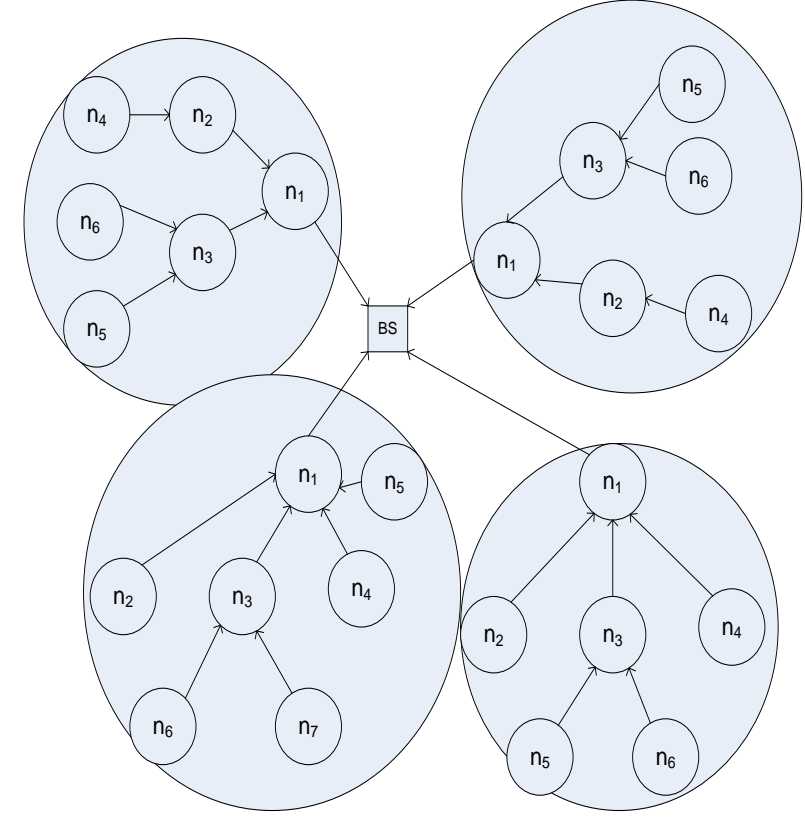

Fig. 3: Task Result Collection Process

\subsection{ETAUC algorithm}

Step.1 The Base Station, BS Allocates the task to each clusters on the basis of their remaining Battery Power $\left(\mathrm{R}_{\mathrm{BC}}\right)$.The $\mathrm{BS}$ assign the task to cluster through their Cluster Head, $H$. The task Assigned to the cluster $T_{C}$ is given as $\mathrm{T}_{\mathrm{c}}=\mathrm{T}_{\text {total }} \times \frac{\mathrm{R}_{\mathrm{BC}}}{100}-\alpha$. Here $\alpha$ is a constant, it depend upon the battery utilization in communication.

Step: 2 The Cluster head, $\mathrm{H}$ collect the information for the remaining battery of their child nodes; assign the task according to their remaining battery power $\left(\mathrm{R}_{\mathrm{NC}}\right)$, the task assigned to a child node is calculated as

$\mathrm{T}_{\mathrm{NC}}=\mathrm{T}_{\mathrm{c}} \times \frac{\mathrm{R}_{\mathrm{NC}}}{100}-\alpha$

The task assigned to the $\mathrm{H}$ is given as

$$
\mathrm{T}_{\mathrm{H}}=\mathrm{T}_{\text {total }} \times \frac{\mathrm{R}_{\mathrm{BH}}}{100}-\beta
$$

$\mathrm{R}_{\mathrm{BH}}$ is the remaining battery power of $\mathrm{H}, \beta>T_{c} \times \frac{R_{N C}}{100}-\alpha$ 
Step 3: Now the child node of $\mathrm{H}$ assigns the task to their child node and itself according to their remaining battery power by using equation (1) and (2).

Step 4: Repeat Step-3 until the leaf node of the DAG does not come.

Step 5: After the distribution of $\mathrm{T}_{\text {total }}$ in the cluster the parent nodes collect the result from their child nodes.

Step 6: Each parent node wait for a threshold time for getting the result from the child node. Suppose the throughput of the processor of the nodes is $\eta$ and the allocated task to the node is $\mathrm{T}_{\mathrm{NC}}$ then the threshold time $(\tau)$.

$\tau=\frac{\mathrm{T}_{\mathrm{NC}}}{\eta}+\lambda+\psi, \lambda$ is the communication cost and $\psi$ is the task partition cost.

\subsubsection{Formation of the relation between temperature and battery backup}

The depletion rate of the battery depends upon the temperature, As we know that WSN is applied in area of varying temperatures and tactical fields. The resultant battery of the cluster can change as the temperature of the cluster change, so it is required to formulize the relation between temperature and battery. If we are able to predict the battery backup of the network, then on the basis of battery backup, we can conclude that the particular work can be done by the network or not. On the basis of remaining battery and varying temperature in the environment, we can get the depletion rate at that particular area. According to this depletion rate we can assign the work to the cluster headas $T_{H}=T_{\text {total }} \times \frac{R_{B H}}{100}-\beta-$ $\omega$, if the depletion rate is greater as compared to the normal depletion rate. The value of constant $\omega$ depends upon the depletion rate of the battery. We have tried to find out the dependency between the temperature and battery backup, for the purpose of establishing the relation, we have applied the linear regression using R-language. The line of regression is given as $r=\phi+\Theta s$, where $\phi$ is intercept and $\Theta$ is coefficient. Using linear regression we have got the $\phi$ and $\Theta$. The relation between temperature(TEMP) and battery backup(BB) can be given as TEMP $=19.1848+0.5527 \mathrm{BB}$. The below given fig. 4 and fig. 5 represent the distribution of Battery Backup(Voltage) and temperature.

\section{Histogram of Voltage}

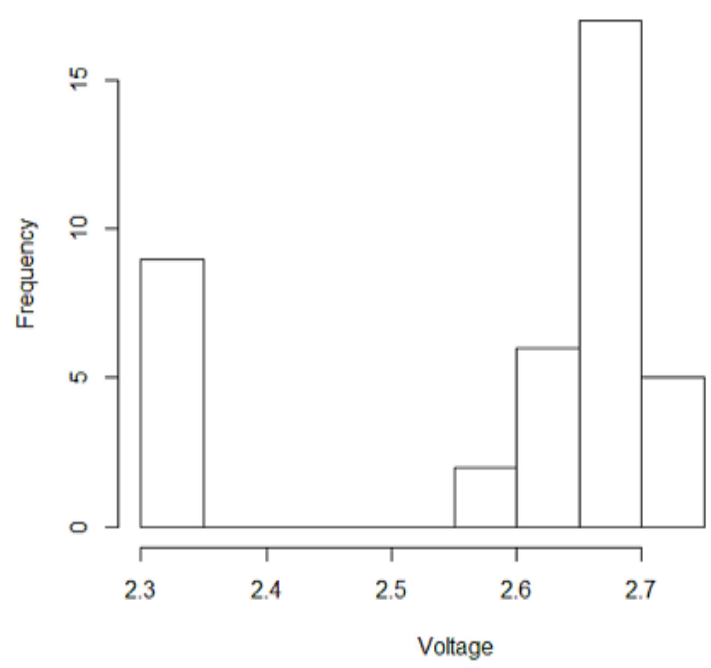

Fig. 4 Distribution of Voltage (Battery Backup)

Histogram of Temperature

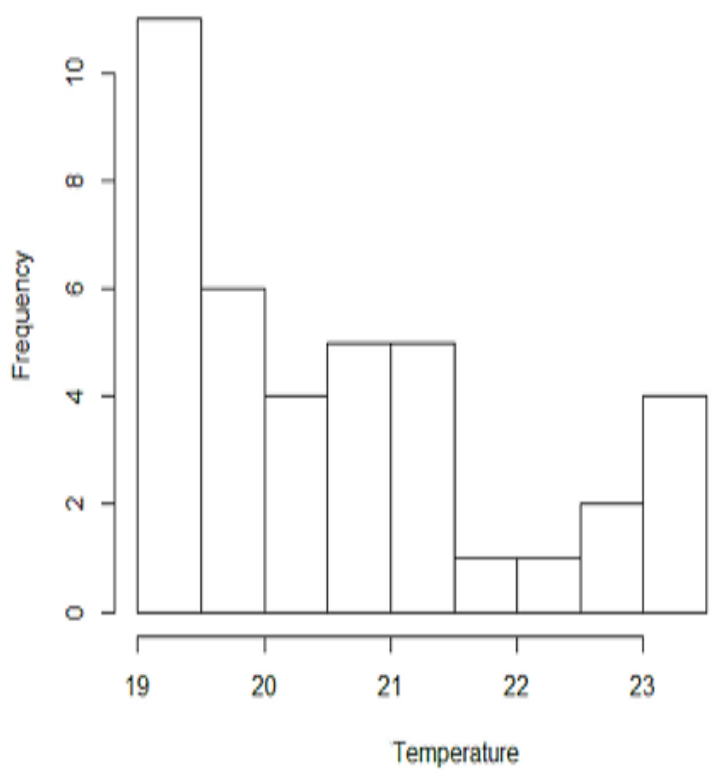

Fig. 5 Distribution of Temperature

\section{SIMULATION RESULTS}

This paper considers the metric for evaluating proposed method as throughput. Before presenting results, we present the simulation model as: There are 64 sensor nodes arrange in 5 clusters with the battery backups of the clusters are 0.51 , $0.21,0.36,0.14$ and 0.04 joules per sec. The simulation model is run for 600 seconds to evaluate the proposed method.Figure 6 shows the throughput of the network without applying the ETAUC and figure 7 represent the throughput of the network after the application of the ETAUC. The results provided as the throughput without application of ETAUC as 6957.4125 bps and after the application of ETAUC as $9219.1683 \mathrm{bps}$. As the result, ETAUC enhance the throughput as $32.51 \%$. The 


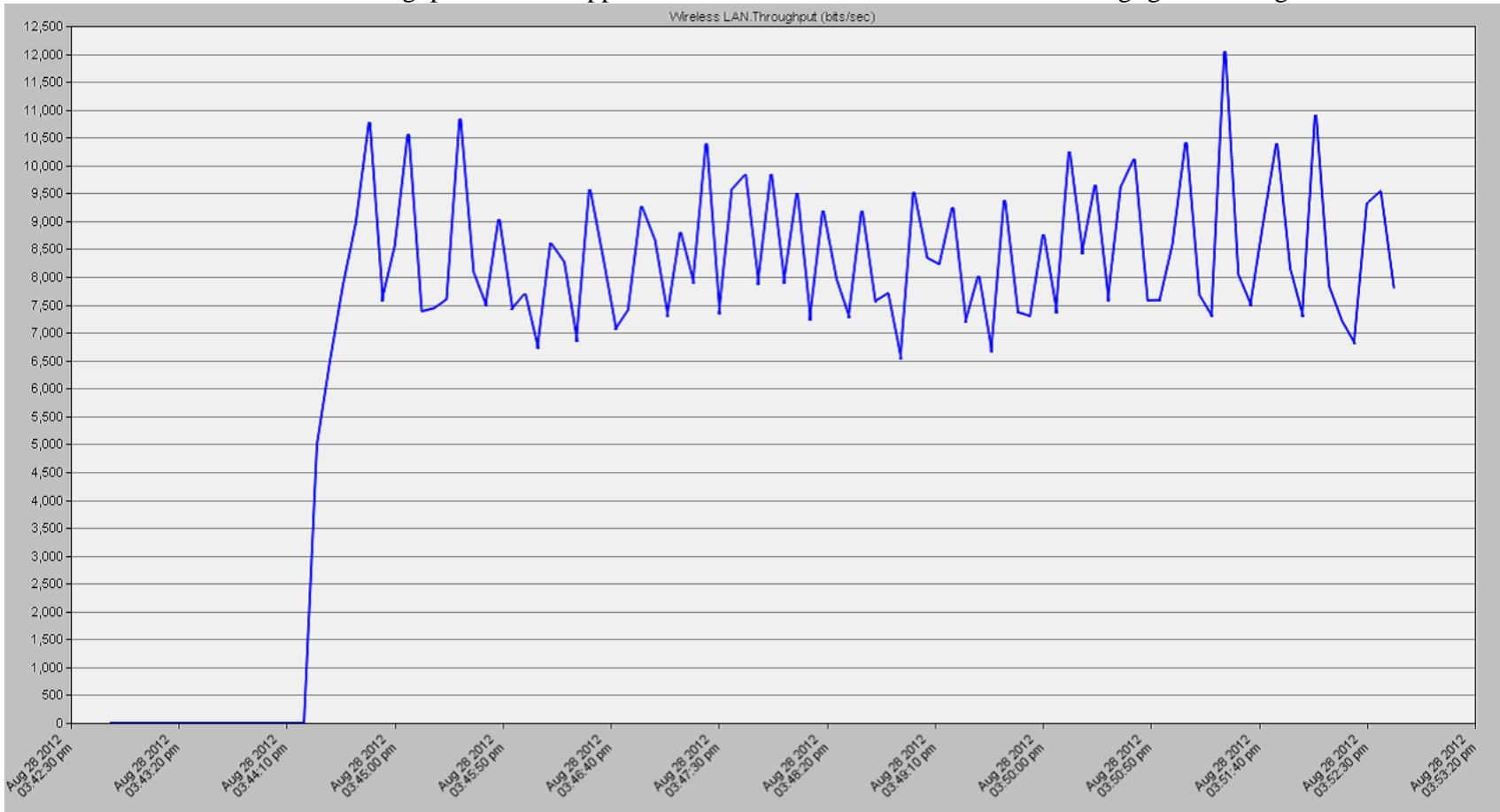

Fig 6: Throughput before the application of ETAUC

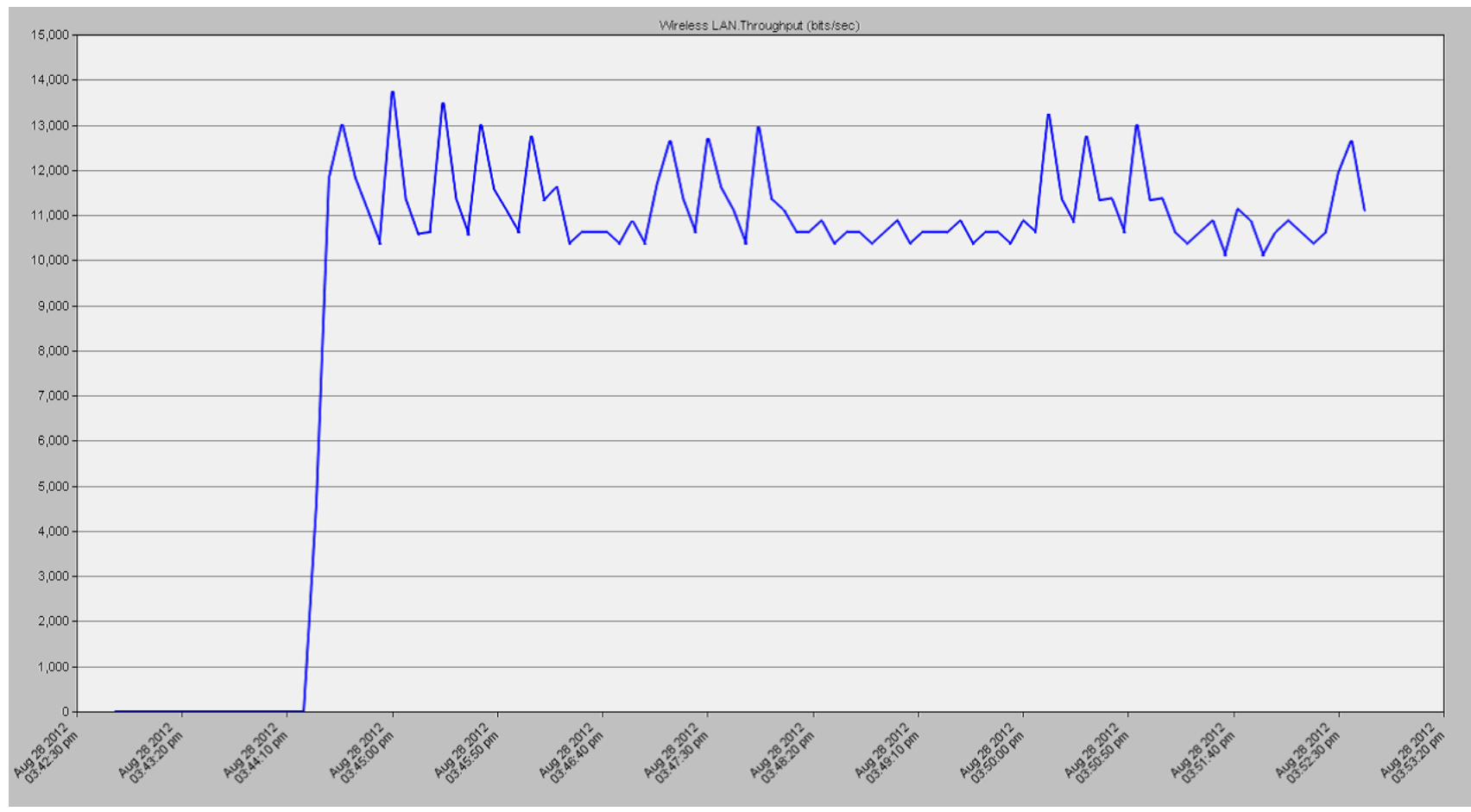

Fig 7: Throughput after the application of ETAUC

\section{CONCLUSION}

This paper presented ETAUC, a method for energy aware task allocation with unequal clustering in wireless sensor network. The aim of this paper is to complete the task given to the network within the lifetime of the network, enhance connectivity and remove the situation in which many sensor nodes get destroyed due to the depletion of battery backup. As we know that sensor network are applied in disastrous area with varying temperature, this paper get focused on establishing the relationship between battery backup and temperature. The proposed method provides $32.51 \%$ increment in the throughput of the network. The future work 
can be done on the automatic selection of cluster head that maintains the efficiency of the ETAUC.

\section{REFERENCES}

[1] I.F.Akyidiz,W.Su,Y. Sankarasubramaniam, and E.Cayirci, "Wireless Sensor Networks: A Survey," Elsevier Computer Networks Journal, vol. 38, no. 4, pp. 393-422, Mar. 2002

[2] D. Estrin, L. Girod, G. Pottie, and M. Srivastava 2001. Instrumenting the World with Wireless Sensor Networks. Proc. IEEE Int'l Conf. Acoustics, Speech, and Signal Processing, pp. 2033-2036.

[3] W. Alsalih, S. Akl, and H. Hassancin 2005. EnergyAware Task Scheduling: Towards Enabling Mobile Computing over Manets. Proc. 19th IEEE Int'l Parallel and Distributed Processing Symp.

[4] C. Meesookho, S. Narayanan, and C.S. Raghavendra 2002. Collaborative Classification Applications in Sensor Networks. Proc. Second IEEE Sensor Array and Multichannel Signal Processing Workshop, pp. 370-374.

[5] A.B. Olsen, F.H.P. Fitzek, and P. Koch 2005, Energy Aware Computing in Cooperative Wireless Networks. Proc. Int'l Conf. Wireless Networks, Comm. and Mobile Computing, pp. 16-21.

[6] M. Singh and V.K. Prasanna 2003. A Hierarchical Model for Distributed Collaborative Computation in Wireless Sensor Networks. Proc. 17th IEEE Int'l Parallel and Distributed Processing Symp.

[7] M. Younis, M. Youssef, and K. Arisha 2002. EnergyAware Routing in Cluster-Based Sensor Networks. Proc. 10th IEEE Int'l Symp. Modeling, Analysis and Simulation of Computer and Telecomm. Systems, pp. 129-136.

[8] K. Akkaya and M. Younis 2003. An Energy-Aware QoS Routing Protocol for Wireless Sensor Networks. Proc. 23rd Int'l Conf. Distributed Computing Systems, pp. 710-715.

[9] X. Lu, H. Hassanein, and S. Akl 2005. Energy-Aware Dynamic Task Allocation in Mobile Ad Hoc Networks. Proc. Int'l Conf. Wireless Networks, Comm. and Mobile Computing, pp. 534-539.

[10] S. Mohanty and V.K. Prasanna 2003. A Hierarchical Approach for Energy Efficient Application Design Using Heterogeneous Embedded Systems. Proc. Int'l Conf. Compilers, Architecture and Synthesis for Embedded Systems, pp. 243-254.

[11] S. Park, V. Raghunathan, and M.B. Srivastava 2003. Energy Efficiency and Fairness Tradeoffs in MultiResource Multi-Tasking Embedded Systems. Proc. ACM Int'l Symp. Low Power Electronics and Design, pp. 469474.

[12] Weiping Liao,LeiHe,Lepak,.M.,"Temperature and

supply Voltage aware performanceand power modeling at microarchitecture level, "IEEE Transations on computer-aided design of Integrated Circuits and Systems, vol. 24, no. 7, July 2005.

[13] D. Estrin, L. Girod, G. Pottie, and M. Srivastava 2001. Instrumenting the World with Wireless Sensor Networks.Int'l Conf. Acoustics, Speech and Signal Processing (ICASSP 2001).
[14] Y. Tian, and E. Ekici, "Cross-Layer Collaborative InNetwork Processing in Multihop Wireless Sensor Networks," IEEE Transactions on Mobile Computing, vol.6, no.3, pp.297-310, March 2007

[15] S. Abdelhak, S. Ghosh, J. Tessier, and M. Bayoumi 2008. Resource and Energy-aware Distributed Blockbased LU Decomposition on Wireless Sensor Networks. High Performance Embedded Computing at Lincoln Labs, pp. 52-53.

[16] S. Abdelhak, R. Chaudhuri, S. Ghosh, and M. Bayoumi 2009. QR Decomposition: Demonstration of Distributed Computing on Wireless Sensor Networks. High Performance Embedded Computing at Lincoln Labs.

[17] J. Luo and N.K. Jha 2000. Power-Conscious Joint Scheduling of Periodic Task Graphs and Aperiodic Tasks in Distributed Real- Time Embedded Systems. Proc. IEEE/ACM Int'l Conf. Computer Aided Design, pp. 357 364.

[18] Abdelhak, S., Gurram, C.S., Ghosh, S., Bayoumi, M. 2010. Energy-balancing task allocation on wireless sensor networks for extending the lifetime. In: Proceedings of IEEE International 53rd Midwest Symposium on Circuits and Systems, Seattle, pp. 781784.

[19] Peter Bodik, Wei Hong, Carlos Guestrin, Sam Madden, Mark Paskin, and RomainThibaux 2004. Intel Lab Data, Intel Berkeley Research Lab. http://db.csail.mit.edu/labdata /labdata.html.

[20] Y. Yu and V.K. Prasanna 2005. Energy-Balanced Task Allocation for Collaborative Processing in Wireless Sensor Networks. Mobile Networks and Applications, vol. 10, pp. 115-131.

[21] Y. Yu and V. K. Prasanna 2003. Energy-balanced task allocation for collaborative processing in wireless sensor networks. ACM SIGPLAN conference on Language, compiler, and tool for embedded systems, pp. 265-274.

[22] T. Xie, X. Qin, and M. Nijim 2006. Solving energylatency dilemma: task allocation for parallel applications in heterogeneous embedded systems. 2006 International Conference on Parallel Processing (ICPP'06).

[23] T. Xie, X. Qin,“An Energy-Delay Tunable Task Allocation Strategy for Collaborative Applications in Networked Embedded Systems," IEEE Transactions on Computers, pp. 329-343, 2008

[24] J. Zhu, J. Li, H. Gao 2007. Tasks Allocation for RealTime Applications in Heterogeneous Sensor Networks for Energy Minimization. Eighth ACIS International Conference on Software Engineering, Artificial Intelligence, Networking, and Parallel/Distributed Computing (SNPD 2007), pp.20-25.

[25] S.Giannecchini, M.Caccamo and C.-S.Shih 2004 Collaborative resource allocation in wireless sensor networks.in Proc. of Euro micro Conference on RealTime Systems (ECRTS'04), pp. 35-44.

[26] P. Basu, W. Ke, and T. D. C. Little, "Dynamic taskbased anycasting in mobile ad hoc networks," Mobile Networks and Applications, vol. 8, no. 5, pp. 593-612, Oct. 2003

[27] Zhang, J. Lin, C. K.Tham, "Energy-efficient adaptive sensor scheduling for target tracking in wireless sensor networks", J Control Theory Appl, vol. 8 no. 1, pp. 86-92 2010. 